\title{
Extension de la sécheresse exceptionnelle observée en 1958 dans les régions équatoriales
}

\section{Extension of the exceptional drought observed in equatorial regions in 1958}

\author{
PAR J. RODIER

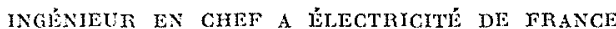 \\ GHFF DU SERVICE HYDROLOGIQUE DE L'OFFICE DE LA RECHERGHE SCIENTIFIQUE ET TECHNIQUE OUTRE-MYER
}

\author{
(Communication présentée par $M$. ROCHE, ingénieur hydrologue à \\ Électricité de France, \\ en l'absence de M. RODIER, excusé.)
}

\begin{abstract}
Etendue de la sécheresse qui a affecté l'Afrique équatoriale en 1958, sur une bande parallèle à l'équateur depuis le Libéria jusqu'à la région des grands lacs. Extension aux régions équatoriales d'Amérique du Sud. Relevés des hauteurs de précipitations et des débits dans chacun des deux hémisphères : le semestre le plas sec correspond $\dot{a}$ la saison des pluies principale et la fréquence d'une telle sécheresse est au maximum décennale; par endroits, elle est cinquantenaire.

Intérêt, pour les études hydrologiques, des conséquences pratiques d'un tel phénomène, s'il présentait un caractère général dans ces régions (exemple des régions soudanaises où il est courant).
\end{abstract}

On a observé depuis longtemps qu'en zone tropicale de l'Afrique de l'Ouest, les périodes de sćcheresse ou d'abondance exceptionnelle intéressent de très vastes superficies le long de bandes parallèles à l'équateur : sécheresse de 1913 et de 1941-1945, abondance de 1955. De telles constatations permettent de pallier, dans une certaine mesure, la carence d'observations hydrologiques pendant de Iongues périodes sur de nombreux cours d'eau, en rendant possible les corrections d'hydraulicité basées sur les observations effectuées pendant de longues périodes à quelques rares stations.

Dans la bande équatoriale, les régimes sont plus complexes et les caractéristiques varient beaucoup suivant la longitude; Jes observations s'étendent en général sur des périodes plus courtes. La complexité des phénomènes généraux commandant le régime fait que la généralisation des sécheresses ou des années abondantes est moins nette. Il a cependant été pos-
The extent of the 1958 arought, which affected a band of Equatorial Africa parallel to equator, from Liberia to the region of the great lakes. Extension of the drought to equatorial regions of South America. Precipitation and runoff recordings in both hemispheres, showing that the driest six months occured during the principal rainy season. and that the frequency of such a drought is at most ten-yearly, and even fifty-yearly in some places.

If this phenomenon were general for the above regions (it is frequently observed in the Sudan. for instance), its practical consequences would be valuable for hydrological surveys.

sible de suivre la sécheresse de 1958, particulièrement sévère, sur de grandes superficies pour procéder à une analyse plus précise. L'année a été fractionnée en deux périodes de six mois contenant chacune une saison des pluies. Dans un premier stade, la sécheresse a été caractérisée par les rapports de pluviosité aux différentes stations. Dans un second stade, on s'est efforcé de déterminer les rapports d'hydraulicité.

\section{Etude des précipitations.}

La carte $n^{\circ} 1$ qui représente les courbes d'égales pluviosités (précipitations en 1958 sur précipitations moyennes de la période) montre qu'au cours du premier semestre le littoral de la Côte d'Tvoire, celui du Dahomey et du Togo sont légèrement excédentaires. A l'intérieur, on note un léger déficit, en général inférieur à $20 \%$.

Au Cameroun, les montagnes de l'ouest pré- 


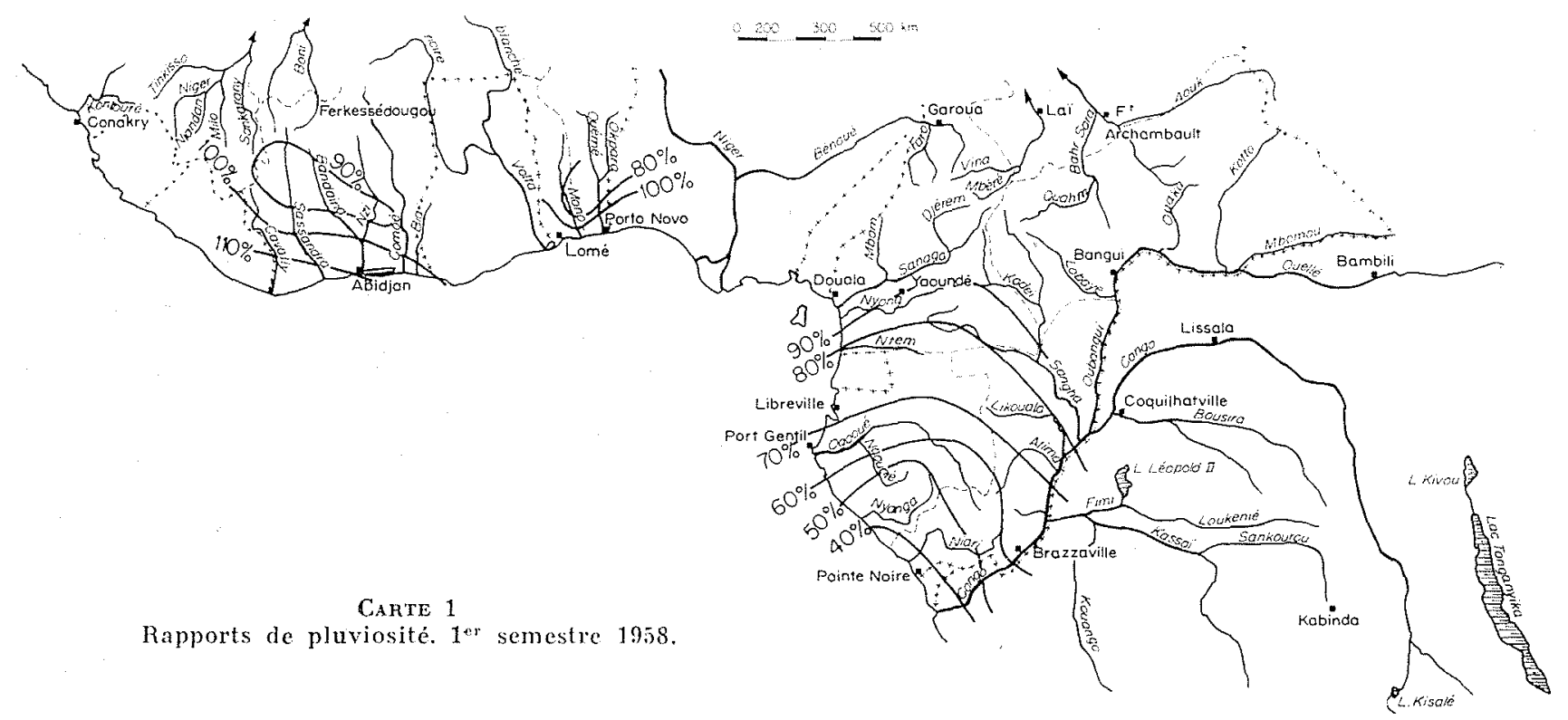

sentent une pluviosité normale, mais on trouve un déficit croissant vers le sud ( $80 \%$ pour le bassin du Nyong). Il semble que ce soit au Gabon que passe la courbe $70 \%$. Le déficit s'accentue encore lorsqu'on descend plus au sud: $50 \%$ sur le bassin du Kouilou. Le littoral présente une sécheresse encore plus accentuée (pluviosité de $18 \%$ à Pointe-Noire). Le déficit s'étend largement sur la partie occidentale du bassin du Congo, le Kasaï en particulier.

On trouve donc pour ce semestre une pluviosité moyenne ou légèrement déficitaire dans l'hémisphère nord, alors que le déficit est très important dans l'hémisphère austral : pratiquement pas de saison des pluies dans certaines régions.

La carte 2 montre la répartition de la pluviosité au cours du second semestre. La Côte
d'Ivoire, et surtout le 'Togo et le Dahomey, accusent un déficit extrêmement sévère : $40 \%$ dans la région de Lomé. Ce déficit disparaît au niveau du dixième parallèle nord. Au Cameroun, la pluviosité ne semble pas descendre en-dessous de $70 \%$. Sur le bassin de l'Ogoué, au Gabon, la pluviosité varie de $65-70 \%$ vers la côte à 80 $90 \%$ à l'est du bassin. La République du Congo présente des déficits importants surtout sur le littoral (Pointe-Noice $35 \%$ ). A l'intérieur, les déficits sont moins marqués ( 70 à $90 \%$ au Congo ex-belge) : les précipitations sont déficitaires au centre et à peu près normales au sud et au nord (Ouellé). Cependant, si l'étiage du dernier trimestre a été très sévère dans cette région, il arrive souvent que décembre ait été très arrosé (Pointe-Noire).

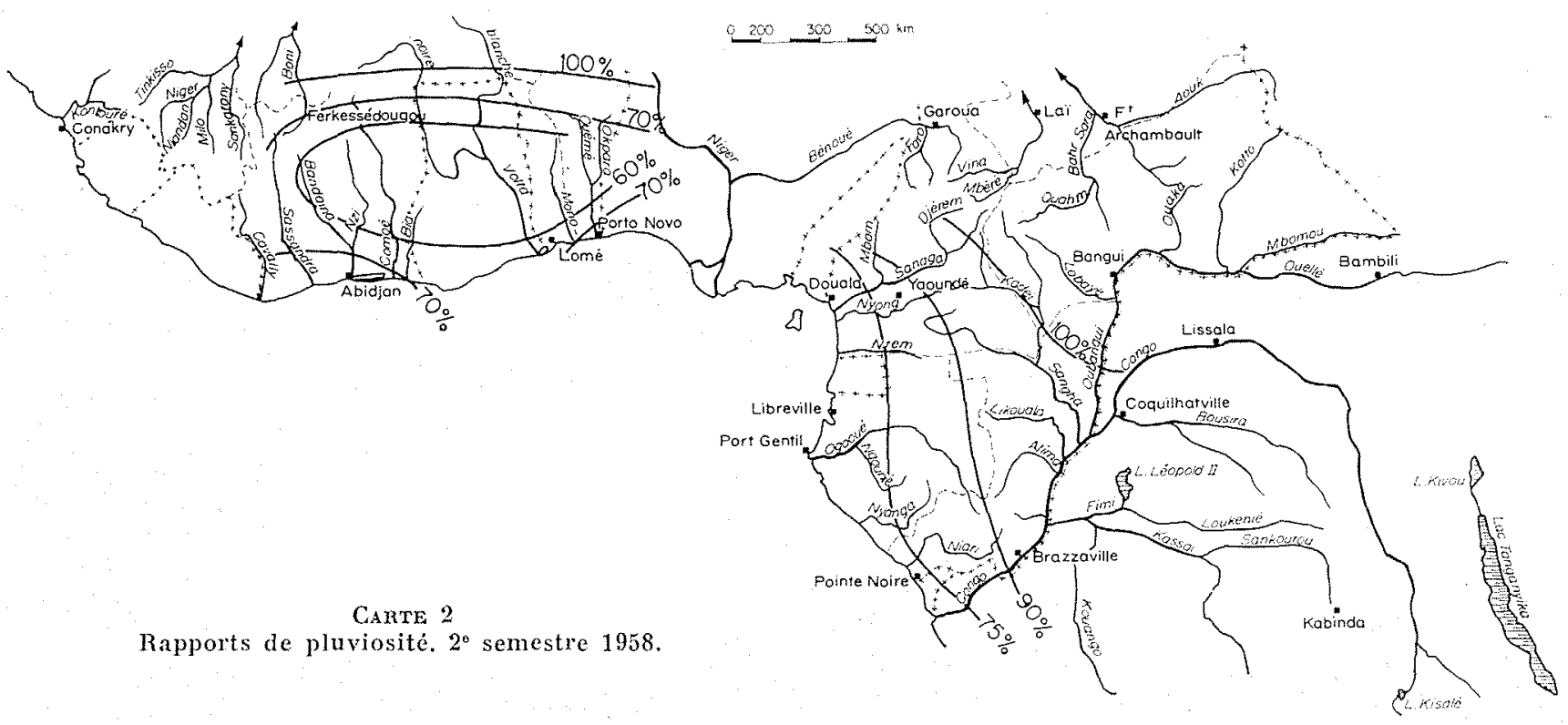




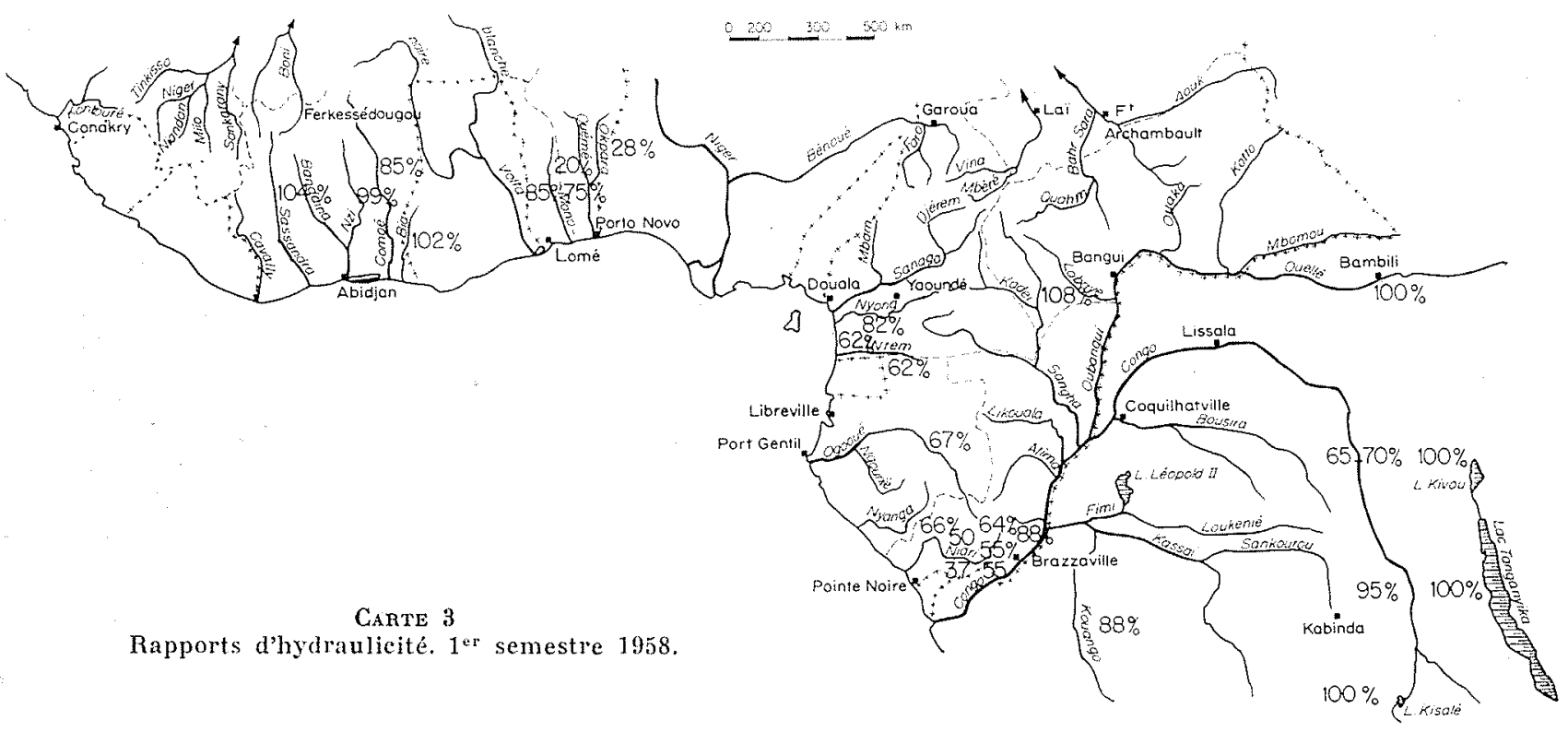

\section{Etude des débits.}

Les rapports d'hydraulicité ont été présentés sur les cartes 3 et 4 . Etant donné la faible densité du réseau, il eût été peu significatif de tracer des courbes d'égales hydraulicités.

Au premier semestre, alors que l'hydraulicité est sensiblement normale dans l'hémisphère nord, sauf au Togo et au Dahomey, où on atteint des valeurs de 20 à $30 \%$, le déficit est très important sous l'équateur (62\%). Dans la bande équatoriale de l'hémisphère austral, la zone déficitaire s'étend vers l'est jusqu'à la région des grands lacs. Elle est limitée au sud par le lac Kisale. D'après les stations de Ponthierville et de Kindou sur la Loualaba, l'hydraulicité au centre du bassin du Congo aurait été de 65-70\%.
Entre Ogooué et Congo, l'hydraulicité doit être comprise entre 50 et $65 \%$ avec, dans les zones les moins arrosées, des valeurs sans doute inférieures à $10 \%$.

Au sud-ouest de la cuvette congolaise, le Kasaï présente une hydraulicité de 85 à $90 \%$.

Entre le deuxième degré de latitude nord et la ligne joignant Usumbura au bassin supérieur du Kasaï et, peut-être, le vingt-huitième degré de longitude est, la fréquence d'une telle sécheresse est partout faible. Pour l'Ogooué, il semble que la période de retour soit d'une trentaine d'années. Malgré les conditions favorables que présente pour le fleuve Congo la grande étendue de son bassin, il semble que les diagrammes de cette période soient de fréquence décennale sèche.

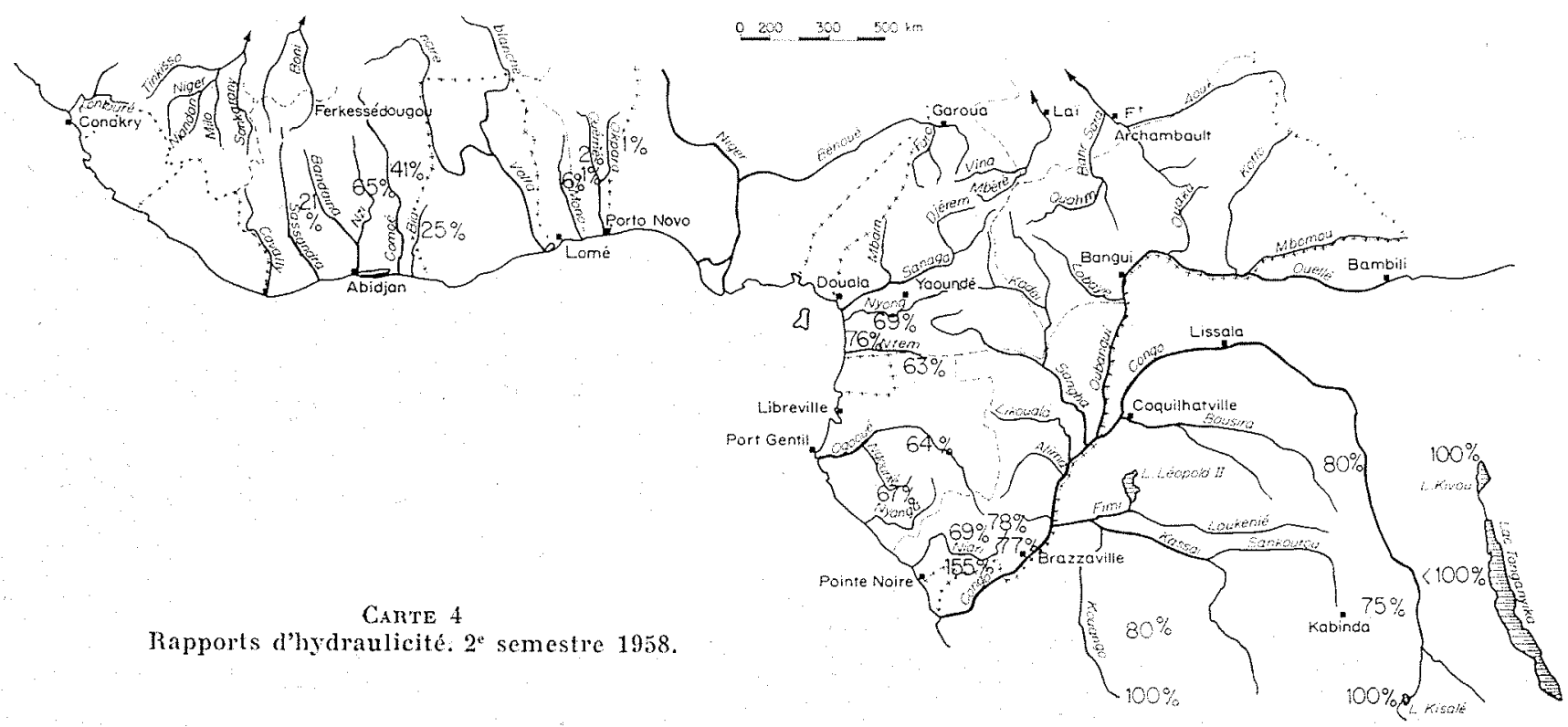


Au cours du second semestre, la sécheresse est extrèmement rigoureuse dans les zones équatoriales de l'hémisphère nord: hydraulicité de 20 à $25 \%$ en Còte d'Ivoire, pouvant s'abaisser à $10 \%$ à l'est; au Togo et au Dahomey, 1 à $2 \%$ sont, durant cette période, les valeurs courantes de l'hydraulicité. A l'ouest et au sud du Cameroun, l'hydraulicité est de l'ordre de $70 \%$, ce qui est faible pour cette région très arrosée. Dans la cuvette congolaise, on observe un peu partout les mêmes tendances : étiage extrêmement bas, de fréquence très inférieure à la fréquence décennale. Les limites de la zone déficitaire sont marquées par le sud du bassin du Kasaï, le sud du bassin de la Loualaba et la région des lacs, quoique le déficit semble déborder sur le lac Tanganyika.

En conclusion, on se trouve bien devant un phénomène de la mème ampleur que ceux qui ont été constatés dans les régions tropicales. Cette même sécheresse exceptionnelle a été observée en Guyane, où I'hydraulicité du premier semestre 1958 varie de 55 à $65 \%$ suivant les bassins et celle du second semestre de 25 à $40 \%$. En Colombie l'hydraulicité a été de l'ordre de 50 à $60 \%$ pour l'ensemble de l'année.

L'intérêt pratique de telles recherches est grand. La principale cause d'imprécision dans l'évaluation des débits moyens mensuels vrais est l'incertitude qui règne sur le rapport entre les moyennes sur les périodes dont on dispose et les moyennes sur de longues périodes. L'existence de séries d'années fortes ou faibles s'étendant sur toute la bande équatoriale permettrait de supposer que l'allure générale des variations d'hydraulicité reste, dans les régions d'Afrique équatoriales, la même que pour de rares stations observées depuis très longtemps. D'autre part, si sécheresse ou abondance commençaient systématiquement dans l'hémisphère sud, il y aurait là un élément précieux de prévision à longue échéance. Pour le vérifier, il faut tout mettre en œuvre pour assurer longtemps la continuité des observations, ce qui n'est déjà pas facile, et attendre le retour de phénomènes exceptionnels de ce genre, en souhaitant toutefois qu'ils n'aient pas de conséquences trop catastrophiques.

D I S CUS SIO N

Président: Durwaut

M. le Président remercie M. Roche pour son exposé et ouvre la discussion.

M. Raud fait la remarque suivante :

« L’année hydrologicue 1957-1958 a été en Algérie wne année tout a fait exceptionnelle, mais surtout par l'ampleur des précipitations de l'automme et du début de l'hiver. Nous avons l'habitude de considérer les années hydrologiques en partant du $1^{\text {er }}$ septembre. Il $y$ a eu de très fortes pluies en octobre, novembre, décembre 1957 et jusqu'au 18 janvier 1958, pluies dont l'intensité a une période de retour sensiblement cincuantenaire sans doute. Par contre, à cette période de très fortes précipitations succédèrent brusquement une fin d'hiver et un début de printemps (fin février) peu pluvieux et le temps devint même subitement très sec à partir de la fin d'avril. Le contraste entre les fortes pluies de la première partie de l'amnée hydrologique et la sécheresse de la fin de la même année a été très marqué. »

«M. Raud ne prétend pas qu'il y ait une relation avec les phénomènes que M. Roche vient de signaler pour l'Afrique équatoriale; sa remarque avait seulement pour but de signaler que l'année $1957-1958$ avait été exceptionnelle en Algérie aussi.

M. Roche dit que cette sécheresse n'a pas affecté particulièrement les régions tropicales. Des endroits placés sous l'influence intertropicale se sont trouvés particulièrement abondants pendant la mème période. La configuration, donnée par M. Raud, du climat d'Algérie est, en un peu plus accentué, celle trouvée aux Antilles à la mème époque.

M. de Rouviles ne pense pas qu'il y ait beaucoup de rapport entre le climat de l'Afrique du Nord et celui de la zone tropicale et demande à M. Rocrry si l'on a essayé de se rendre compte de l'époque de l'année où s'est produite la sécheresse. Dans la zone tropicale, les précipitations ont liet un mois après le passage du soleil au zénith : or, il y passe deux fois par an, alors qu'en Algérie il n'y passe pas. Est-ce dans la période intersolaire qu'il y a eu le plus srand déficit?

D'autre part, M. de Rovville n'est pas sû́ qu'il existe une similitude entre le climat des Antilles et celui de la zone tropicale; les latitudes se rapprochent assez; mais il peut $y$ avoir une cause autre que le phénomène solaire dans les précipilations de celle zone. M. Rovrer s'est-il rapproché de la Météorologie à ce sujet-lá?

M. Roche répond que les observations météorologiques faites dans ces pays tropicaux et équatoriaux étant succincles, il ne peut répondre a ces questions.

M. le Président demande à M. Roche s'il a des données sur les régions plus proches des régions considérées, comme le sud du Soudan on le Kenya, et sur le Brésil.

M. Roche dit que les régions du Soudan et du Renya ont été sensiblement normales, car elles se trouvent plutôt dans un climat tropical. De plus, all Kenya, le climat. est un pew particulier du fait des influences orographiques. M. Roche a montré sur la carte que la limite du deficit d'hydraulicité était indiquée par la zóne des grands lacs, c'est-à-dire l'ouest du Kenya. Ni le Kenya, ni le sud du Soudan n'ont été affectés par cette sécheresse.

Au Brésil, on entre dans les climats tropicaux du sud. M. Rocres a cité le cas de la Colombie qui est un test pour l'ensemble des zones équatoriales d'Amérique. Il est probable que le nord du Brésil, s'il est sous l'influence équatoriale, a été déficitaire. La sécheresse de 1950 était strictement limitée aux zones équatoriales.

M. Roche ajoute que le phénomène qu'a décrit M. Ravo pour l'Afrique du Nord ne doit pas avoir de liaison avec la sécheresse équatoriale. Du reste, cela ne porte pas sur la même période, puisqu'il s'agissait pour l'Afrique du Nord de l'année hydrologique 19571958 , alors que M. Rocme a considéré l'année calendaire 1958.

M. Ravb dit que la sécheresse a commencé à partix du deuxieme trimestre 1958. Il y a eu une inversion très marquée, car, auparavant, c'était très humide.

M. Roche dit que, dans ce cas, cela correspondrait aux périodes observées au Toggo et au Dahomey.

M. le Président remercie M. Rocine en le priant de transmette les remerciements de la S.H.F. à M. Rodrer. 